\title{
The Impact of Human Microbiota in Ocular Inflammation
}

\author{
Pedro Simões* \\ Egas Moniz Hospital, Portugal \\ *Corresponding author: Pedro Simões, Egas Moniz Hospital, Rua da Junqueira, 126, Lisbon, Portugal
}

Submission: 眥 May 13, 2018; Published: 㘹 May 15, 2018

\begin{abstract}
Microbiota corresponds to the microbial associates that exist in the human body, and the genes they encode, the microbiome. Recent advances in genetic techniques are contributing to a better microbiota characterization. Imbalance in the intestinal microbiota - dysbiosis - is associated with several pathologies and reveals its important role as immunomodulator. These developments are particularly significant in ocular immunology, revealing physiopathologic mechanisms and identifying potential therapeutic targets.
\end{abstract}

\section{Introduction}

An immeasurable amount of commensal microbes colonize the skin and mucosal surfaces. Together, the microbial associates that exist in the human body constitute the microbiota, and the genes they encode, the microbiome (Table1) [1]. Referred to as our "forgotten organ", the microbiota appears to play a major

Table 1: Terms commonly used in the study of the microbiota [25]

\begin{tabular}{|c|c|}
\hline Term & Meaning \\
\hline Dysbiosis & Microbial imbalance \\
\hline Enterotype & Stratification based on the intestinal microbiota \\
\hline Microbiome & Collection of the genomes of the microorganisms found on the host \\
\hline Microbiota & Community of microorganisms found on the host \\
\hline Probiotic & Dietary supplement containing live beneficial bacteria \\
\hline
\end{tabular}

\section{Factors Affecting Normal Microflora}

Commensal microflora or microbiota consists of the microorganisms present on body surfaces covered by epithelial cells. specific conditions they can overcome protective host responses and exert pathologic effects [4]. Several factors are known to favor the growth of relatively aggressive resident bacteria at the expense of beneficial commensals, leading to dysbiosis. Among them are aging, diet, antibiotic use patterns, exercise, smoking, public health measures and infections [5-7].

\section{Intestinal Permeability and the Microbiota}

Dysbiosis seems to modify intestinal mucosal permeability. The interaction between intestinal microbiota and the mucosal These micro-organisms co-evolve with their hosts, however, under role in health and disease [2]. Recent advances in next generation sequencing (NGS) technology have enabled to characterize the human microbiota, with unprecedented resolution, and to identify possible related etiopathogenic mechanisms [3]. In this review, the authors give an overview of the current understanding of the microbiota and its potential impact on ocular health. wall is mediated by the same receptors which can recruit effector Th cells and activate innate immunity ensuing inflammation [8]. Zonulin modulates the permeability of intestinal tight junctions and consequently is a marker for enhanced intestinal permeability. Lamprecht, et al. [9] showed that probiotic supplementation decreased Zonulin is feces adding more information on the dysbiosis role on increased intestinal permeability [9]. Accordingly, increased intestinal permeability is associated with autoimmune disease including ankylosing spondylitis, multiple sclerosis and autoimmune hepatitis [10-12].

\section{Microbiota and Systemic Autoimmune Disease}

It is theorized that dysbiosis may facilitate autoimmunity at both barrier sites and internal organs. Complex interactions 
between genetics, environment, and the microbiota outline the inflammatory status and this encroaches autoinflammatory and autoimmune diseases [13]. Several animal models support a connection between intestinal bacteria and arthritis. On the one hand, germ-free rats developed severe joint inflammation in an adjuvant-induced arthritis model when compared to conventionally raised controls, suggesting that the gut microbiota may have important immunosuppressive effects [13]. On the other hand, it was reported the proarthritogenic role of Bacteroides species when introduced into germ-free arthritis-prone rats [14] (Figure 1).

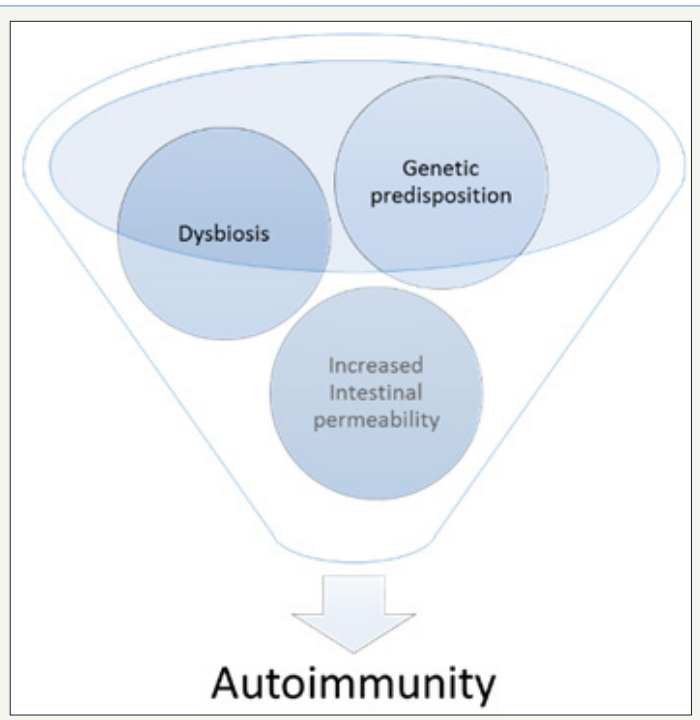

Figure 1: Proposed role of microbiota in autoimmune diseases.

These data illustrate the dysbiosis role in triggering autoimmunity. Genetic susceptibility combined with increased intestinal permeability and dysbiosis, appear to increase the propensity of antigen-presenting cells to become activated and present antigen to cognate $\mathrm{T}$ cells, in secondary lymphoid organs, leading to the development of autoreactive lymphocytes [15]. Autoimmunity typically wanes over time [16]. Thus, it is appealing to speculate that relapses could be linked to the intricate relations where microbiota interrelates. Generally, a diverse microbiota is a sign of intestinal health. Restricted diversity emerges as a mark of a variety of disease states including autoimmunity $[17,18]$. Interestingly, a dysbiosis characteristic signature was found in patients with Behçet disease [19].

\section{The Microbiota and Ocular Disease}

Most models of autoimmune uveitis require inoculation with some retinal antigen in adjuvant to trigger disease. Horai et al. [20] developed a mouse model of spontaneous uveitis in that activation of retina-specific $\mathrm{T}$ cells was dependent on intestinal commensal microbiota, hypothesizing, that activation of autoreactive $\mathrm{T}$ cell receptors by commensal microbes could be a common trigger of uveitis, enlightening the paradox of how spontaneous autoimmunity can occur in an immune privileged site. In a model of experimental autoimmune uveitis, Nakamura et al. [21] suggested a protective and, equally, potentially prouveitogenic, intestinal microbiota.
In this study, the severity of the uveitis was modulated with oral antibiotics raising the idea of using a short course of antibiotics followed by repopulation of the intestine with beneficial species to mitigate disease severity.

\section{Ocular Surface Microbiota}

Most of the studies evaluating ocular surface microbiota have been performed with older technology that could not access for accurate biodiversity. However, more recent studies with NGS technology, found that healthy ocular surface microbiota is paucibacterial when compared to adjacent skin and different mucosa [22]. Greater bacterial counts are present in contact lens wear and dry eye disease [23-24].

\section{Conclusion}

There is increasing evidence that the microbiota has potent immunoregulatory functions, consequently, investigation with respect to ocular inflammatory diseases is wide open and auspicious.

\section{References}

1. Clemente JC, Ursell LK, Parfrey LW, Knight R (2012) The impact of the gut microbiota on human health: an integrative view. Cell 148(6): 12581270.

2. O'Hara, A M and F Shanahan (2006) The gut flora as a forgotten organ. EMBO Rep 7(7): 688-693.

3. Jovel J, Jordan Patterson, Weiwei Wang, Naomi Hotte, Sandra O'Keefe, et al. (2016) Characterization of the Gut Microbiome Using 16 S or Shotgun Metagenomics. Front Microbiol 7: 459.

4. Tlaskalova-Hogenova H, Stepánková R, Hudcovic T, Tucková L, Cukrowska B, et al. (2004) Commensal bacteria (normal microflora), mucosal immunity and chronic inflammatory and autoimmune diseases. Immunol Lett 93(2-3): 97-108.

5. Sartor RB (2001) Intestinal microflora in human and experimental inflammatory bowel disease. Curr Opin Gastroenterol 17(4): 324-330.

6. Wu GD, Chen J, Hoffmann C, Bittinger K, Chen YY, et al. (2011) Linking long-term dietary patterns with gut microbial enterotypes. Science 334(6052): 105-108.

7. Luc Biedermann, Jonas Zeitz, Jessica Mwinyi,Eveline Sutter-Minder, Ateequr Rehman, et al. (2013) Smoking cessation induces profound changes in the composition of the intestinal microbiota in humans. PLoS One 8(3): e59260.

8. Marasco G, Di Biase AR, Schiumerini R, Eusebi LH, Iughetti L, et al. (2016) Gut Microbiota and Celiac Disease. Dig Dis Sci 61(6): 1461-1472.

9. Lamprecht M, Bogner S, Schippinger G, Steinbauer K, Fankhauser F, et al. (2012) Probiotic supplementation affects markers of intestinal barrier, oxidation, and inflammation in trained men; a randomized, doubleblinded, placebo-controlled trial. J Int Soc Sports Nutr 9(1): 45.

10. Buscarinu MC, Cerasoli B, Annibali V, Policano C, Lionetto L, et al. (2017) Altered intestinal permeability in patients with relapsing-remitting multiple sclerosis: A pilot study. Mult Scler 23(3): 442-446.

11. Lin R, Zhou L, Zhang J, Wang B (2015) Abnormal intestinal permeability and microbiota in patients with autoimmune hepatitis. Int J Clin Exp Pathol 8(5): 5153-5160.

12. Martinez-Gonzalez O, Cantero-Hinojosa J, Paule-Sastre P, Gómez-Magán JC, Salvatierra-Ríos (1994) Intestinal permeability in patients with ankylosing spondylitis and their healthy relatives. Br J Rheumatol 33(7): 644-647. 
13. Kohashi O, Kuwata J, Umehara K, Uemura F, Takahashi T, et al. (1979) Susceptibility to adjuvant-induced arthritis among germfree, specificpathogen-free, and conventional rats. Infect Immun 26(3): 791-794.

14. Rath HC, Herfarth HH, Ikeda JS, Grenther WB, Hamm TE, et al. (1996) Normal luminal bacteria, especially Bacteroides species, mediate chronic colitis, gastritis, and arthritis in HLA-B27/human beta2 microglobulin transgenic rats. J Clin Invest 98(4): 945-953.

15. Ruff WE, MA Kriegel (2015) Autoimmune host-microbiota interactions at barrier sites and beyond. Trends Mol Med 21(4): 233-244.

16. Wildner G, U Kaufmann (2013) What causes relapses of autoimmune diseases? The etiological role of autoreactive T cells. Autoimmun Rev 12(11): 1070-1075.

17. Cho I, MJ Blaser (2012) The human microbiome: at the interface of health and disease. Nat Rev Genet 13(4): 260-270.

18. Scher JU, Ubeda C, Artacho A, Attur M, Isaac S, et al. (2015) Decreased bacterial diversity characterizes the altered gut microbiota in patients with psoriatic arthritis, resembling dysbiosis in inflammatory bowel disease. Arthritis Rheumatol 67(1): 128-139.

19. Consolandi C, Turroni S, Emmi G, Severgnini M, Fiori J, et al. (2015) Behcet's syndrome patients exhibit specific microbiome signature. Autoimmun Rev 14(4): 269-276.
20. Horai R Zárate-Bladés CR, Dillenburg-Pilla P, Chen J, Kielczewski JL, et al. (2015) Microbiota-Dependent Activation of an Autoreactive T Cell Receptor Provokes Autoimmunity in an Immunologically Privileged Site. Immunity 43(2): 343-353.

21. Nakamura YK, Christina Metea, Lisa Karstens, Mark Asquith, Henry Gruner, et al. (2016) Gut Microbial Alterations Associated With Protection From Autoimmune Uveitis. Invest Ophthalmol Vis Sci 57(8): 3747-3758.

22. Doan T, Akileswaran L, Andersen D, Johnson B, Ko N et al. (2016) Paucibacterial Microbiome and Resident DNA Virome of the Healthy Conjunctiva. Invest Ophthalmol Vis Sci 57(13): 5116-5126.

23. de Paiva CS, Dan B Jones, Michael E Stern, Fang Bian, Quianta L Moore, et al. (2016) Altered Mucosal Microbiome Diversity and Disease Severity in Sjogren Syndrome. Sci Rep 6: 23561.

24. Zhang H, Zhao F, Hutchinson DS, Sun W, Ajami NJ, et al. (2017) Conjunctival Microbiome Changes Associated With Soft Contact Lens and Orthokeratology Lens Wearing. Invest Ophthalmol Vis Sci 58(1): 128-136.

25. Rosser EC, C Mauri (2016) A clinical update on the significance of the gut microbiota in systemic autoimmunity. J Autoimmun 74: 85-93. (c) (i) Creative Commons Attribution 4.0

International License

For possible submissions Click Here
Submit Article

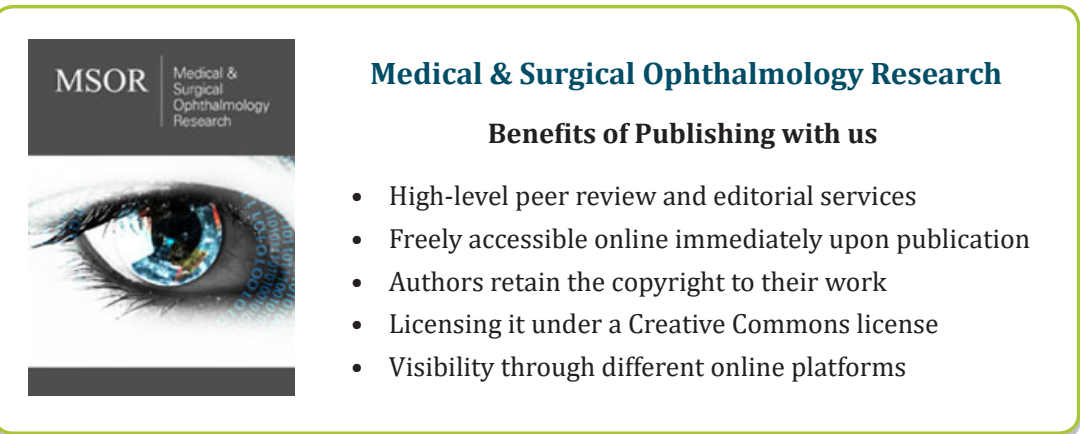

